\title{
Alpha spectrometry with the inexpensive open-source detector Alphaino
}

\author{
Luca Gugliermetti $^{\mathrm{a}}$, Luigi Lepore ${ }^{\mathrm{b}, *}$, Romolo Remetti ${ }^{\mathrm{b}}$, Massimiliano Colarieti \\ Tosti ${ }^{c, d}$ \\ ${ }^{a}$ Sapienza - University of Rome, Department of Astronautics Engineering, Electrical and \\ Energy, Via Eudossiana, 18 - 00184, Rome - Italy \\ ${ }^{b}$ Sapienza - University of Rome, Department of Basic and Applied Sciences for \\ Engineering, Via Antonio Scarpa, 14 - 00161, Rome - Italy \\ ${ }^{c} K T H$ - Royal Institute of Stockholm, Department of Medical Engineering, Hälsovägen 11C, \\ SE-14157 Huddinge - Sweden \\ ${ }^{d}$ Karolinska Institutet, Department of Clinical Science, Intervention and Technology \\ (CLINTEC), SE-17177, Stockholm - Sweden
}

\begin{abstract}
A photodiode-based inexpensive detector working as counter and spectrometer for alpha particles, named Alphaino, is described and analyzed in depth. Prior to detector construction, Monte Carlo simulations by means of MCNPX ver. 2.7.0 code have been carried out to select the most suitable sensitive element for the intended applications. The detector has been tested for low rate alphaparticle counting and spectroscopy, demonstrating a maximum achievable count rate of $4 \cdot 10^{3} \mathrm{~s}^{-1}$, with an energy resolution corresponding to a Full Width Half Maximum of $160 \mathrm{keV}$ over the entire energy range of measured alpha, namely $4-6.5 \mathrm{MeV}$, the intrinsic efficiency being 100\%. Alphaino can be used for fast and qualitative analyses to radionuclides identification, and quantitative analyses when radionuclides monitored are characterized by well distinguished energy lines in spectra. Its applications will include ${ }^{222} \mathrm{Rn}$ progeny monitoring by mechanical air sampling on a millipore filter, and disposable applications to qualitative measuring of potentially alpha-contaminated liquids (containing plutonium isotopes) by a droplet put on the photodiode itself, for a quick and
\end{abstract}

\footnotetext{
* Corresponding author

Email addresses: luca.gugliermetti@uniroma1.it (Luca Gugliermetti), luigi.lepore@uniroma1.it (Luigi Lepore), romolo.remetti@uniroma1.it (Romolo Remetti), massimiliano.colarieti-tosti@sth.kth.se (Massimiliano Colarieti Tostic,)
} 
cheap evaluation prior to initiate proper chemical treatments to classical alpha spectrometry by electrodeposition. Alphaino is completely open-source. The repository for the project is available on GITHUB at https://github.com/ bemxgm/Radon-Monitor.

Keywords: Alpha spectrometry, Photodiode, MCNPX, Open-Source

\section{Introduction}

Alphaino is an alpha particles detector intended for applications where low cost and small dimension are key factors. It can be used both as an alpha particles counter, and for alpha spectroscopy. Photodiodes are highly suitable for

5 alpha detection and many photodiode-based detectors are available. Some examples with excellent energy resolution and a wide range of count rate capability are given in the following. J.E. Bateman [1] developed a scintillator-photodiode detector suitable for the detection of high energy charged particles. R.J. Meijer [2] developed a detector for alpha particles $(13-20 \mathrm{MeV})$ and protons $(3-12$

${ }_{10} \mathrm{MeV}$ ) with a large area photodiode coupled to a plastic scintillator. T. Streil [3] developed a low-cost RAM alpha detector for radon monitoring (5.5 - 7.9 $\mathrm{MeV})$. A. Ahmed [4] developed a CMOS custom integrated circuit for detecting alpha particles. P. Ashokkumar [5] developed a ${ }^{222} \mathrm{Rn} /{ }^{220} \mathrm{Rn}$ targeted alpha detector using a silicon PIN diode inside a high voltage electrostatic precipitation

15 chamber, to monitor radon and thoron concentrations by spectral analysis of detected alphas.

The low-cost Alphaino detector here presented uses a photodiode as the sensitive part, without the need of any scintillation element coupled. Alpha particles impinge the photodiode directly releasing their energy in the active volume. The energy resolution achieved, Full Width Half Maximum of $160 \mathrm{keV}$ over the entire energy range of measured alpha spectra, allows for a mathematical deconvolution and reconstruction of each nuclide activity, overcoming the intrinsic limitations of gross counting or peaks overlapping in complex spectra. Excellent performances are obtained in intrinsic efficiency. Alphaino is thought 


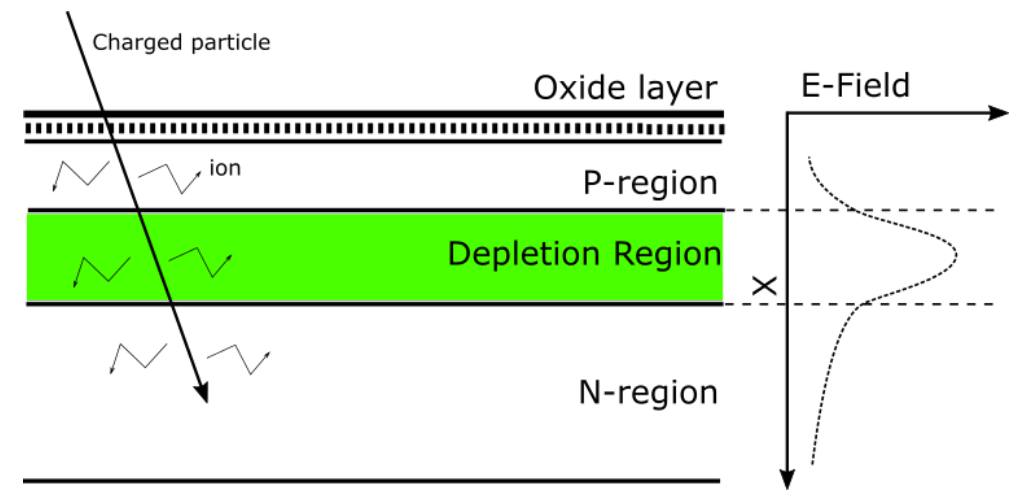

Figure 1: Charge collecting process and associated electric field (E-Field).

25 for cheapness and easiness: with respect to similar detectors [4] [5], no vacuum chambers or high voltage for electrostatic precipitation toward the sensor are implemented, and alpha sources are placed in front of the photodiode directly. Foreseen applications include: i) ${ }^{222} \mathrm{Rn}$ progeny monitoring by mechanical air sampling on a millipore filter; ii) qualitative measuring of potentially alphacontaminated liquids (containing plutonium isotopes for instance) by putting a droplet on the photodiode itself, for a quick and cheap evaluation prior to initiate longer and expensive chemical treatments used in classical alpha spectrometry by electrodeposition.

In alpha detection, charge collection can be achieved even with standard commercial silicon photodiodes. Interacting with silicon, the alpha generates electric charges proportional to the energy deposited by the particle. Alphas in silicon have a significant Linear Energy Transfer (LET), which allows the deposition of almost all the energy in just the photodiode's depletion region, Figure 1. As a consequence, the amount of charge collected at photodiode's 40 pins is strongly correlated to the energy with which the alpha particle entered into the silicon layer that acts as the sensitive volume. The current signal is then converted into a voltage pulse whose maximum amplitude is proportional to the energy deposited by the alpha particle.

Even though the electric signal generated by a typical $5 \mathrm{MeV}$ alpha particle 
in silicon is quite large, about ten times stronger than a proton of similar energy and hundreds times stronger than an electron of similar energy, alpha counting and spectroscopy present challenges. Detection efficiency is a complex function of source-detector relative position, and the same applies to the energy deposition expected inside the active volume of the detector. Presence of air between source and detector, or any coating layers give raise to a low-energy cut-off and a low-energy-tail in spectral peaks. Also, any variation in thickness or composition in coating layers over the sensor area or in the surrounding layers of the depletion region can affect the energy resolution.

When monitoring alpha-emitters dispersed in air e.g. radon or thoron, environmental conditions such as humidity or dust dispersed in air could be significant on Alphaino's responses. Moreover, when using photodiodes as sensitive elements, the intrinsic high level of the dark current at room temperature and its dependence with temperature should be considered. Having been thought as part of the modular environment HOPES (Home Pollution Embedded System the effects of variable environmental conditions on Alphaino responses could be mitigated.

Alphaino can also be connected both to traditional nuclear spectroscopy electronics, and to cheap prototyping boards, e.g. Arduino. A web platform, through which the system can be operated, has been already introduced by Gugliermetti et al. in [7].

The paper herein discusses the characterization of Alphaino as an alpha particles spectrometer, allowing to consider such detector a reliable and cheap alternative to traditional alpha spectrometry, or to build a smart radon detector capable to measure radon concentration by correlating its daughters' potential alpha energy concentration in air.

The detector is open-source: everyone can download the schematics, build the sensor and contribute to further development through the GITHUB repository for the project, https://github.com/bemxgm/Radon-Monitor. 


\section{Methods}

Detector Design. The detector is made by Surface Mount Device (SMD) components easily available on the market. The whole cost of the detector is about $35 \$$ at the time when the paper was written. All details about electronics and connections are discussed in Appendix A and available through the GITHUB repository.

The sensitive element is a reverse-biased wide-area photodiode $\left(93.6 \mathrm{~mm}^{2}\right)$ by Silonex [8]. Such photodiode has been found particularly suitable for the application due to its specific features: $i$ ) wide area, intended to be coupled with alpha-sources having an electrodeposited circular surface within $7 \mathrm{~mm}$-indiameter, when the source is lying on the detector and the air gap is minimized; ii) high reliability, allowing to consider the sensor to be heavy duty and stable, ensuring the measurement repeatability; iii) thin oxide passivation layer of the front surface, reducing the energy losses in alpha tracks toward the sensitive volume as more as possible (the coating layer was assumed to be $0.09 \mu \mathrm{m}$ ); iv) 90 low capacitance and high speed, allowing for a quick collection of charges, fast generation of the voltage pulse, and detector recovery.

Prior to the detector construction and verification, the photodiode's response has been studied by means of Monte Carlo simulations in the Monte Carlo NParticles eXtended, MCNPX, code ver. 2.7.0 [9] [10]. The transport equation for radiation is solved in Monte Carlo methods by a random sampling of the probability distribution functions ruling physical phenomena. The reproduction of such physical phenomena happening in real experiment within the simulation can be achieved by the definition of a simulation model the more accurate as possible in terms of geometry and materials (dimensions, isotopic composition

and density). Regarding alpha particles transport, the code's default physics is here used.

Radionuclides in calibrated alpha sources here used are deposited onto stainless steel disc, $25 \mathrm{~mm}$-in-diameter, $0.5 \mathrm{~mm}$ thick, and the deposition diameter is $7 \mathrm{~mm}$. According to the manufacturer [11], reagents used are ultra-pure, result- 
ing in an essentially mass-less deposit. This process minimizes line broadening and produces sources exhibiting line widths of less than $20 \mathrm{keV}$ Full-Width-HalfMaximum, FWHM. So, the deposition can be considered so thin to produce only a little broadening on alpha energy lines; tracks emerging can be assumed as the half of the total activity deposited, while the other half is emitted toward the stainless steel disc.

The source model in MCNPX simulations was calibrated to reproduce real sources emission spectra in order to get a first evaluation of the photodiode response, and select a suitable photodiode. Into the MCNPX code the source has been interpreted as an extended isotropic source (a thin cylinder) emitting monoenergetic alpha-particles at 4.7, 6 and 7.5 MeV: energies selected are theoretical values distributed over a wide range, in order to observe differences in detected peaks. The source model in MCNPX (the source definition card, the sdef) is modeled as non attenuating cylinder, as to preserve the $50 \%$ of the tracks emerging from the front side, and the other half emitted toward the source casing. Each alpha line is broadened by means of a gaussian function centered at the alpha line energy with a FWHM=20 keV. The source spectrum is reported in Figure 2 .

The photodiode sensitive volume has been modeled into MCNPX as a $10 \mathrm{~mm}$ x $10 \mathrm{~mm}$ x $0.4 \mathrm{~mm}$ silicon wafer, covered with a $0.09 \mu \mathrm{m} \mathrm{SiO}_{2}$ layer, both materials at standard densities. The energy deposition in the detector's sensitive volume is scored by means of a tally $f 8$ vs. alpha [10] (the tally is the MCNPX tool to score results). The energy deposition spectrum detected by the Silonex SLCD-61N5 photodiode is reported in Figure 2. For each alpha line, in the geometrical configuration considered i.e. the photodiode in front of the source with an air gap $<0.1 \mathrm{~mm}$, an efficiency value of $49 \%$ is found for each peak. Such a result is found to be reasonable due to the fact that about the half of the total alpha emission is loss in the stainless steel disc while the other is emitted toward the photodiode. Low-energy tails in the spectrum were expected due to the continuous energy loss of alphas in their paths, because a variable amount of energy is deposited in the oxide layer according to each specific track. 


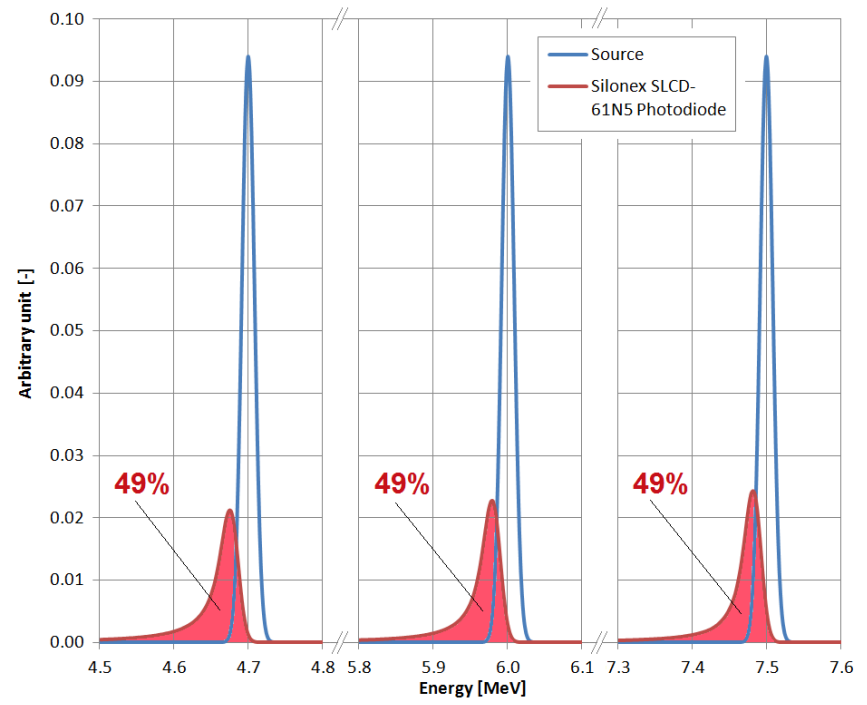

Figure 2: Simulated spectra by MCNPX: gaussian peaks represent alpha particles emerging from the source; asymmetric peaks represent alpha tracks detected by the Silonex SLCD$61 \mathrm{~N} 5$ photodiode when the air gap is $<0.1 \mathrm{~mm}$. Percentages report the total efficiency of the photodiode in the geometrical configuration considered.

Having found in simulation the Silonex SLCD-61N5 photodiode [8] particularly suitable for the intended application, the Alphaino detector has been developed around such component. Outputs from the detector's board can be distinguished in i) a voltage pulse signal whose maximum amplitude is proportional to the energy deposited by the alpha particle; ii) a TTL signal for each alpha detected, for counting purposes only. The whole electronics and schematics are discussed in Appendix A.

A closed environment is also necessary to avoid noise due to the visible light that can reach the photodiode surface, and producing undesired signals. Such precaution can be combined in an electromagnetic-shielded (EMS) dark chamber, able to reduce to light and electronic noise during operations of Alphaino. A 3D printable box can be a solution: Figure 3 shows an example of such box, printed with opaque materials (e.g. black Poly Lactic Acid, PLA, or Acrylonitrile Butadiene Styrene, ABS). The electromagnetic shield can be made by 

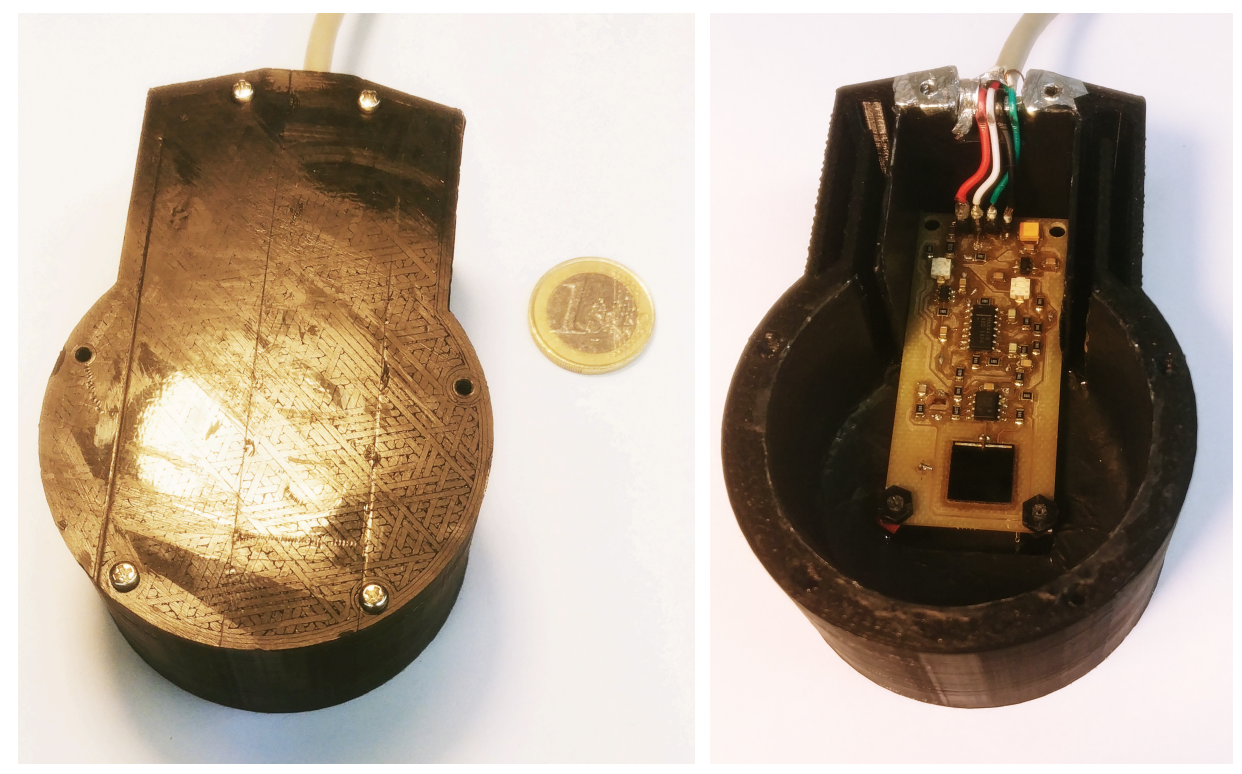

Figure 3: Left: 3D printed case with light labyrinth and dust filters. Right: Alphaino detector inside its ABS box acting as EMS and light shielding.

covering the inside or outside of the box with a conductive layer.

The Alphaino's case in Figure 3 exploits a conductive painting applied on the box interior, acting as an electro-magnetic shield. A light labyrinth with ventilation holes is present on both sides of the box, and a $0.5 \mathrm{~mm}$ nylon net is glued on the ventilation holes to act as dust filter. The choice of using a holed box is due to further works where the detector will be tested in monitoring radon concentration-in-air. All the tests reported in this work were made using such enclosure.

Detector Characterization. The section herein describes all the steps carried out to characterize Alphaino's performances. Different tests have been exploited:

160 Test 1: 'Baseline observation' A direct observation of the baseline voltage. The measurement includes white noise due to electronics. It has been made with a digital multimeter at the moment of switching on, and after 1, 3, $5,7,26$ hours of continuous operation. 
Test 2: 'Stability test' The detector has been coupled to a nuclear amplification system, ORTEC 672 Spectroscopy Amplifier, to obtain voltage output signals between $0-10 \mathrm{~V}$ and then fed to a multi-channel analyzer, ORTEC EASY-MCA 2k. The detector within its enclosure has been exposed to a $2 \mathrm{~mm}$-in-diameter electrodeposited $300 \mathrm{~Bq}{ }^{241} \mathrm{Am}$ check alpha-source placed within a $<0.1 \mathrm{~mm}$ air-gap in front of the photodiode. At least, to assure the repeatability of measurements over time, spectra and count rates have been collected and analyzed vs. time during Alphaino's continuous operation, at detector start-up and after 1, 3, 5, 7, 26 hours of operational activity.

Test 3: 'Detector calibration' Two calibrated $7 \mathrm{~mm}$-in-diameter electrodeposited alpha-sources have been subsequently measured with Alphaino, 'Pu-Am$\mathrm{Cm}$ ' and ' $\mathrm{Np}-\mathrm{Am}-\mathrm{Cm}$ '. Sources were placed over the photodiode, at a distance involving an air gap of $<0.1 \mathrm{~mm}$ between the photodiode and the source surface. The instrumentation used for the test is the same of Test 2. The spectra obtained have been fitted and deconvoluted with the following procedure. After different trials regarding the peak-shape function to spectra deconvolution from [12], the Bortels and Collaers model [13] was found to be the best solution:

$$
\begin{aligned}
& p_{i, j}(u)=N_{j} \cdot b_{i, j} \cdot\left\{\left(\frac{1-\eta}{\tau_{1}}\right) e^{\left(\frac{u-\mu_{i, j}}{\tau_{1}}+\frac{\sigma^{2}}{2 \tau_{1}}\right)} \operatorname{erfc}\left[\frac{1}{\sqrt{2}}\left(\frac{u-\mu_{i, j}}{\sigma}+\frac{\sigma}{\tau_{1}}\right)\right]\right.
\end{aligned}
$$

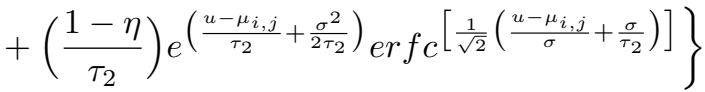

where:

- $u$ is the abscissa of the function: here, channel-unit is considered;

- $p_{i, j}$ is the probability density function describing the monoenergetic $i^{\text {th }}$ alpha-peak shape of the nuclide $j^{\text {th }}$;

- $N_{j}$ is an arbitrary constant for the nuclide $j^{\text {th }}$ considered, to be determined via least-square fit; 
- $b_{i, j}$ is the branching ratio of the $i^{t h}$ alpha-line of the nuclide $j^{\text {th }}$ considered;

- $\mu_{i, j}$ is the nominal energy of the $i^{t h}$ alpha-line of the nuclide $j^{\text {th }}$ considered;

- $\eta, \sigma, \tau_{1}, \tau_{2}$, are parameters describing the peculiar alpha-peak shape from detector, to be determined via least-square fit. Such parameters are fixed and considered valid for each peak in the spectrum;

- erfc is the complementary error function.

Regarding Test 3, the probability density function described in Eq. 1 has been implemented in a Python script [14] in order to perform a least-square deconvolution considering the most significant alpha lines from each nuclide in the spectrum measured. The nominal alpha-emission energies of the nuclides in radioactive sources analyzed have been used to define the channel where the maximum of the $p_{i, j}(u)$ lies, once the energy calibration of the multi-channel analyzer was achieved. The theoretical spectrum is considered as the sum of $p_{i, j}(u)$ curves, one for each alpha-line considered. A least-square fit between measured and simulated spectra has been performed in order to obtain the $N_{j}$ parameters for nuclides involved, and $\eta, \sigma, \tau_{1}, \tau_{2}$ parameters to define the peak-shape function in Eq. 1. With the deconvolution achieved as described above, the correct number of counts in the spectrum for each nuclide has been computed from the sum all $p_{i, j}(u)$ alpha-lines for each nuclide. A comprehensive count rate, $R$, has been then calculated dividing by the acquisition live time. As known, detector efficiency for such comprehensive peak is related to the nominal activity of the nuclide considered by:

$$
R=A \cdot \varepsilon \cdot b
$$

where:

- $R\left[\mathrm{~s}^{-1}\right]$ is the count-rate of the comprehensive peak, namely the sum of all $p_{i, j}(u)$ alpha-lines for the nuclide $j^{\text {th }}$ considered; 

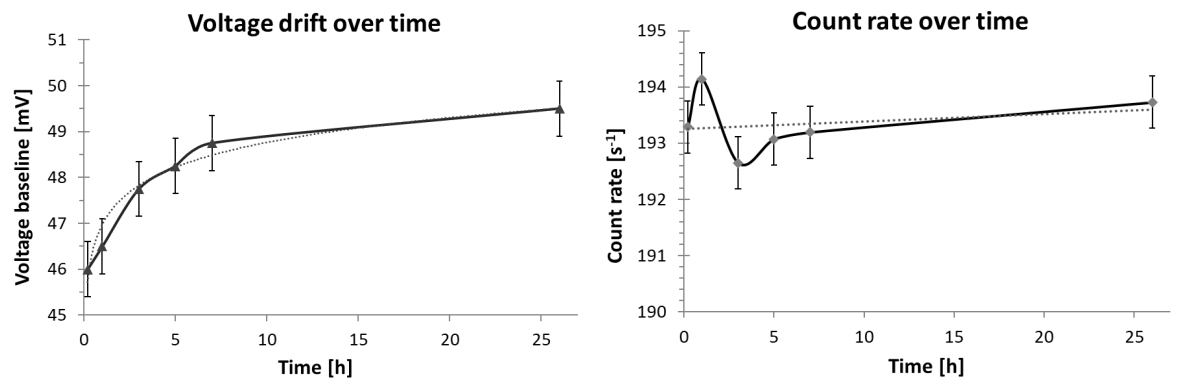

Figure 4: Left: Alphaino's baseline drift collection vs. time when source is not present. Right: Alphaino's count rates drift collection vs. time when alpha source is in position.

- $A[\mathrm{~Bq}]$ is the activity of the nuclide $j^{\text {th }}$ considered;

- $b[-]$ is the comprehensive branching ratio of all alpha-lines considered for the nuclide $j^{\text {th }}$ analyzed;

$-\varepsilon[-]$ is the detector efficiency for the cluster of alpha-lines analyzed.

\section{Result and Discussion}

Results of the tests described in $\$$ 五 are reported hereafter.

Regarding Test 1, Figure 4 -left shows how the operating voltage changes over time, from switching on to regime, in absence of alpha sources. The total drift measured with the TENMA 72-7780 digital multimeter is $3.5 \pm 0.3 \mathrm{mV}$, the RMS noise is $6 \pm 1 \mathrm{mV}$, and the full-scale $46 \pm 1 \mathrm{mV}$. The total electronic read-out uncertainty can be approximated to $11 \mathrm{mV}$; the base voltage drift due to dark current, is about $46 \mathrm{mV}$. Voltage drift can be considered negligible if compared to normal maximum operational voltage of $2.5 \mathrm{~V}$. By considering the useful signal (from a ${ }^{241} \mathrm{Am}$ source) amplified to be set at $1.25 \mathrm{~V}$ the half of the maximum voltage allowed for the pulses- and a comprehensive noise of $11 \mathrm{mV}$, a signal-to-noise ratio $>100$ can be calculated in detecting alpha particles.

Regarding Test 2, Figure 4 -right shows how the count rate, in presence of a constant alpha source, changes over time. A change of 2 counts per second, about the $0.24 \%$ of the average count rate, was observed during the first 26 


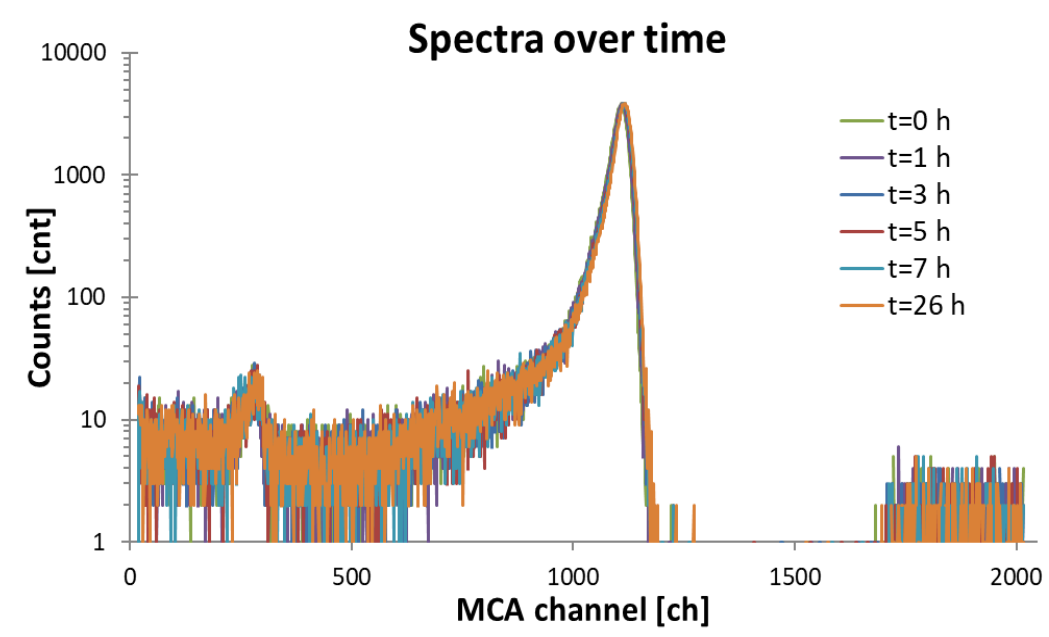

Figure 5: Repetition of the Alphaino's measured spectrum vs. continuous operation time of the detector, when a check alpha source is placed in front of the sensor. Each spectrum has been collected for a 15 min live-time.

235 hours. The stability of the measured spectrum vs. continuous operation time of Alphaino has been evaluated recording several spectra after the detector startup as shown in Figure 5 , each spectrum collected for a 15 min live-time. No differences are observable on the peak's position and shape: the peak recognition can be considered stable i.e. spectra over time are practically overlapping. From the Figure 0 , it is possible to observe the pile-up effect on the bottom right (high channels).

Regarding Test 3 , the spectrum of the ' $\mathrm{Pu}-\mathrm{Am}$ - $\mathrm{Cm}$ ' source has been acquired for a live-time corresponding to $2.7 \mathrm{~h}$. When measuring the ' $\mathrm{Pu}-\mathrm{Am}$ - $\mathrm{Cm}$ ' source, Alphaino recorded a dead-time of $24.1 \%$, the overall count-rate for the spectrum 245 is $\sim 1.7 \cdot 10^{3} \mathrm{~s}^{-1}$. The spectrum of ' $\mathrm{Np}-\mathrm{Am}$ - $\mathrm{Cm}$ ' source has been collected for a live-time corresponding to $1.3 \mathrm{~h}$. The electronics recorded a dead-time of $2.2 \%$, the overall count-rate for the spectrum is $\sim 1.3 \cdot 10^{2} \mathrm{~s}^{-1}$. It should be recalled that the real-time of the measurement can differ from the actual live-time of electronics availability. Such difference is the dead-time, an effect due to electric 250 signals overlapping phenomena, occurring at high count-rates especially. When 


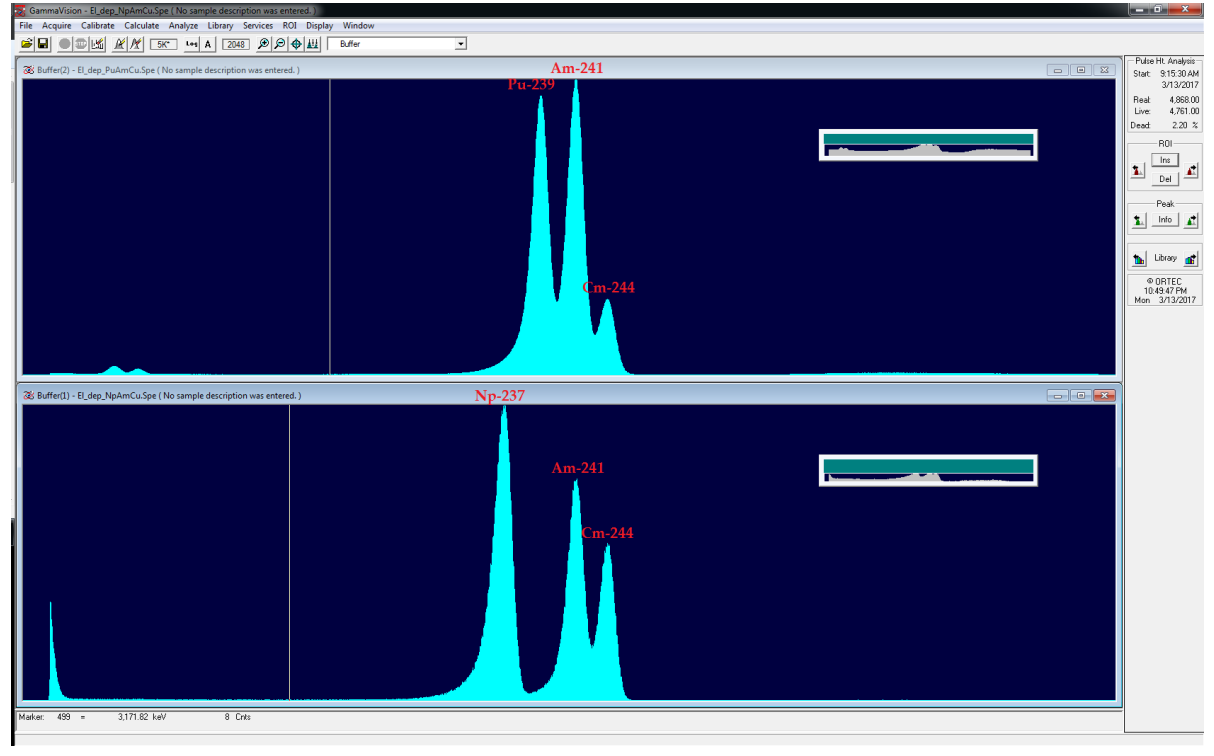

Figure 6: Collected spectra from 'Alphaino' when exposed to calibrated alpha-sources in Table 1 . The upper window is showing the ' $\mathrm{Pu}-\mathrm{Am}-\mathrm{Cm}$ ' source measurement; the lower one is showing the ' $\mathrm{Np}-\mathrm{Am}-\mathrm{Cm}$ ' source measurement. Alpha-clusters from each isotope are flagged in the figure to a quick readout.

a first pulse is detected, a second one too close in time may be ignored because the electronic chain could be blind to such pulse, being busy in evaluating the first one. Figure 6 reports the collected spectra: for a quick readout, alphaclusters are flagged with the corresponding nuclide.

Both reference spectra collected have been used for the energy calibration of the multi-channel analyzer first. The cluster peaks in Figure 6 have been fit with the gaussian least-squares best-fit model, as recommended by §18.V.C.2 of Knoll [15]. Results are reported in Figure 7 for visual evaluation, while numerical results are reported in Table Q: four energy-channel couples are shown, within the energy-calibration model identified. A $\mathrm{R} \sim 0.9998$ value from the linear bestfit model selected ensures Alphaino's good linearity between the primary energy of the alpha particles and the energy depositions collected by the detector itself.

With the energy calibration of the multi-channel analyzer achieved, the deconvolution method in $\S 2$ has been applied to reference spectra in Figure 6 . 

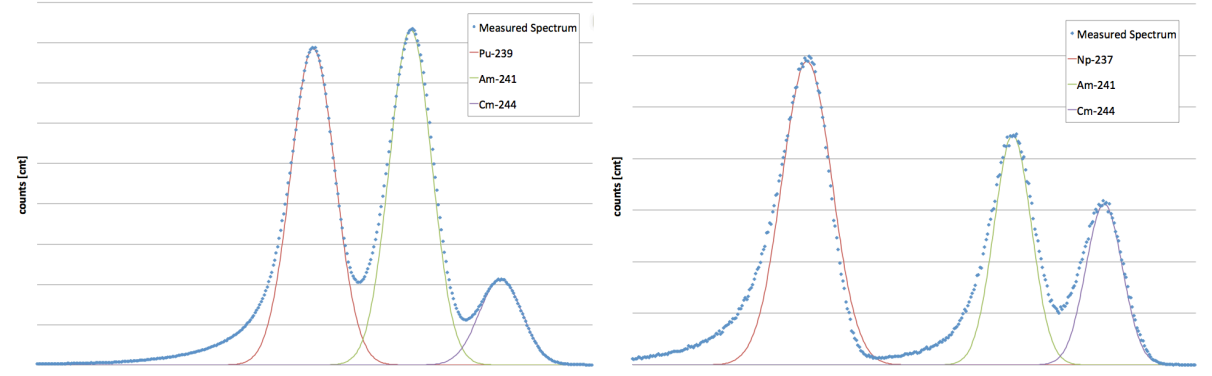

Figure 7: Alpha-cluster peaks from Alphaino' fitted with a Gaussian-model function at their highest part. Left: 'Pu-Am-Cm'. Right: 'Np-Am-Cm'. Channel calculated for Gaussians' centroids have been associated to characteristics $\alpha$-cluster average energy for each nuclide, as reported in Table 1 .

Table 1: Energy couples identified to perform the energy calibration of detector. The panel shows the linear energy calibration model adopted: $\mathrm{R} \sim 0.9998$ ensures the goodness of fitting.

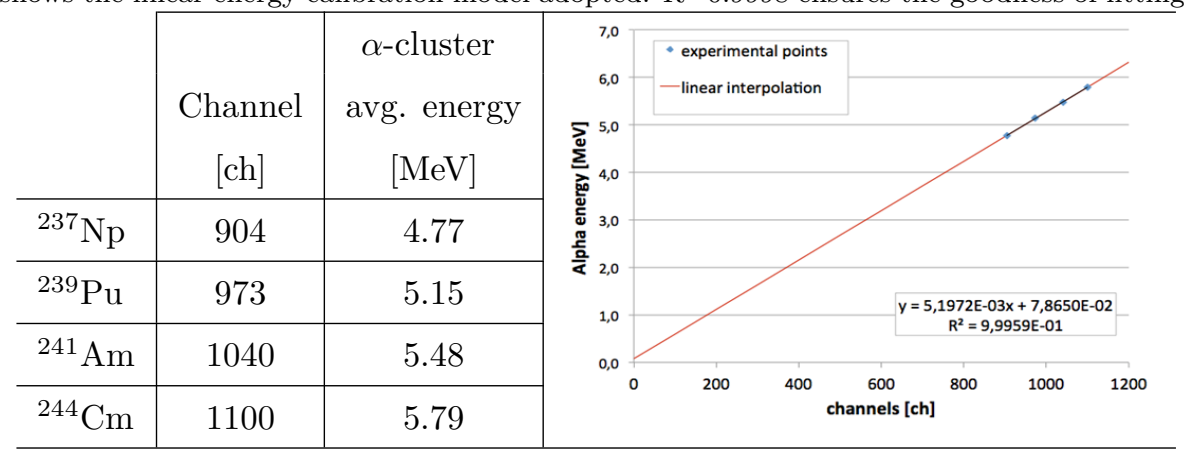



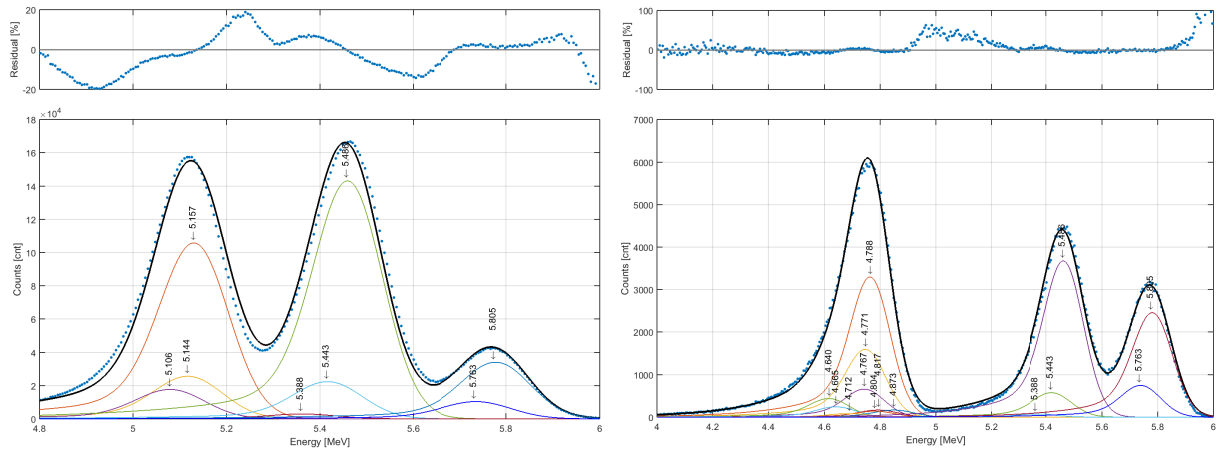

Figure 8: Deconvolution of the alpha spectrum from Alphaino. Continuous lines are the peaks fitting while dotted line is the measured spectrum. Energy tags report each single $\alpha$-energy line contributing to the measured spectrum. Left: 'Pu-Am-Cm'. Right: ' $N p-A m-C m$ '.

Considering a measured spectrum, each alpha-line expected is represented as described in Eq. 1, then such theoretical energy-lines have been summed in a theoretical spectrum to be compared with the measurement. The least-square fit among theoretical and measured data provided the $\eta, \sigma, \tau_{1}, \tau_{2}$ parameters describing the peculiar alpha-peak shape from detector, and the $N_{j}$ value in 270 Eq. 1 for each nuclide. Results of deconvolution method applied to measured spectra are reported in Figure 8. Colored continuous lines shows theoretical alpha-energy emission from each nuclide, being the black the sum of all such theoretical contributes. Dotted lines represent measured spectra. Graphs on the top of the spectra show residuals i.e. the difference between measured counts and theoretical counts, channel by channel. An energy resolution (FWHM) of $160 \mathrm{keV}$ and constant over the entire energy range of detected alpha particles, namely $4-6.5 \mathrm{MeV}$, has been calculated.

With the deconvolution achieved, the efficiency evaluation throughout Eq. has been carried out. Table reports all values in Eq. 6 for measured spectra, nuclide by nuclide. It could be recalled that the count rate for each nuclide, as the comprehensive branching ratio, have been considered as the sum of all contributions from the nuclide considered. Calculated efficiencies are presented in 
Table 2: Overall efficiency calculation for each nuclide in measured spectra analyzed. Branching ratios have been taken from $[16]$.

\begin{tabular}{c|c|c|c|c}
\multicolumn{5}{c}{ Source $\# 1$, 'Pu-Am-Cm' } \\
\hline & $\begin{array}{c}R, \text { count rate } \\
{\left[\mathrm{s}^{-1}\right]}\end{array}$ & $\begin{array}{c}b, \text { branching } \\
{[\%]}\end{array}$ & $\begin{array}{c}A \text {, activity } \\
{[\mathrm{Bq}]}\end{array}$ & $\begin{array}{c}\varepsilon, \text { overall } \\
{[\%]}\end{array}$ \\
\hline${ }^{239} \mathrm{Pu}$ & $826.0 \pm 0.3$ & 99.77 & $1632( \pm 5 \%)$ & $51 \pm 3$ \\
\hline${ }^{241} \mathrm{Am}$ & $933.4 \pm 0.3$ & 99.34 & $1893( \pm 5 \%)$ & $50 \pm 3$ \\
\hline${ }^{244} \mathrm{Cm}$ & $246.0 \pm 0.2$ & 100.00 & $512( \pm 5 \%)$ & $48 \pm 3$ \\
\hline
\end{tabular}

Source \#2, ' $\mathrm{Np}-\mathrm{Am}-\mathrm{Cm}$ '

\begin{tabular}{l|c|c|c|c}
\hline & $\begin{array}{c}R, \text { count rate } \\
{\left[\mathrm{s}^{-1}\right]}\end{array}$ & $\begin{array}{c}b, \text { branching } \\
{[\%]}\end{array}$ & $\begin{array}{c}A, \text { activity } \\
{[\mathrm{Bq}]}\end{array}$ & $\begin{array}{c}\varepsilon, \text { overall } \\
{[\%]}\end{array}$ \\
\hline${ }^{237} \mathrm{~Np}$ & $76.6 \pm 0.1$ & 97.93 & $150( \pm 5 \%)$ & $52 \pm 3$ \\
\hline${ }^{241} \mathrm{Am}$ & $48.8 \pm 0.1$ & 99.34 & $100( \pm 5 \%)$ & $49 \pm 3$ \\
\hline${ }^{244} \mathrm{Cm}$ & $36.2 \pm 0.1$ & 100.00 & $73( \pm 5 \%)$ & $49 \pm 3$ \\
\hline
\end{tabular}

the last column of Table 2 . Uncertainties are calculated by mean of error propagation considering 1- $\sigma$ Poisson statistics on registered counts and uncertainties specified for sources' activities.

As expected, the geometrical configuration used in measurements allows all alpha particles emerging from the sources (about the $50 \%$ of the whole alpha particles created in the source, the other half are emitted toward the source casing) to impinge the photodiode sensor. The stopping power of the detector's coating material cause some energy to be loss for each peculiar alpha track, as shown in low-energy-tail of each emitted alpha energy line. Focusing on a single alpha-energy line, by considering the whole peak in the spectrum, every track is detected and contributes to the counts, so a value of $50 \%$ is expected in efficiency, as confirmed in MCNPX simulations also. Table 2 reports efficiency values for the analyzed nuclides.

In this geometrical configuration where the source is lying in front of the detector with an air-gap $<0.1 \mu \mathrm{m}$, alpha particles impinging the detector are 


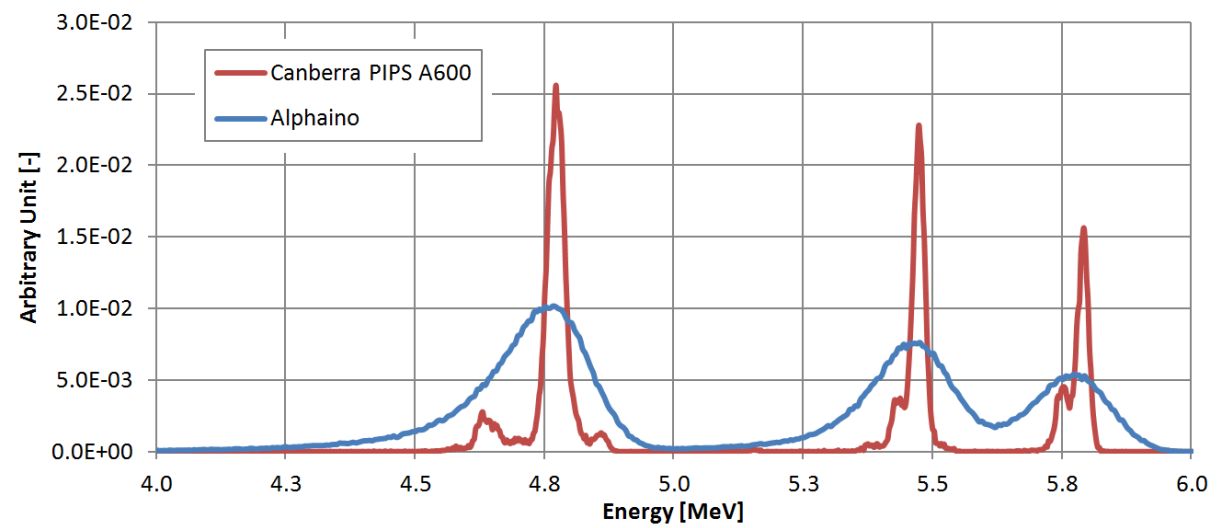

Figure 9: Comparison between experimental spectra acquired by Alphaino and a classical Passivated Implanted Planar Silicon detector, PIPS Canberra A600 when the ' $\mathrm{Np}-\mathrm{Am}-\mathrm{Cm}$ ' source is monitored. Spectra are normalized to the same area. FWHM result in $160 \mathrm{keV}$ and $23 \mathrm{keV}$ for Alphaino and PIPS A600, respectively.

about the half of the total emission from the source, the intrinsic efficiency of Alphaino vs. alpha particles can be considered about $100 \%$.

A comparison between Alphaino and a classical Passivated Implanted Planar Silicon detector, PIPS Canberra A600 [17] is reported, Figure 9. Spectra are normalized to the same area. FWHM results in $160 \mathrm{keV}$ and $23 \mathrm{keV}$ for Alphaino and PIPS A600, respectively.

\section{Conclusion and perspectives}

An open-source and inexpensive detector working as counter and spectrometer for alpha particles has been developed. Details on device's hardware and software can be found on a Github repository https://github.com/bemxgm/ Radon-Monitor, being available through a CC BY-SA creative commons license.

Despite its low cost, Alphaino demonstrated to be suitable as spectrometer and gross-counter for alpha particles. The signal-to-noise ratio is $>100$, the voltage drift is negligible, and the count rate is not affected by significant electronic noise. The sensitive element of the detector is transparent to photons, showing high sensitivity for alpha particles. 
Two calibrated radioactive sources, ' $\mathrm{Pu}-\mathrm{Am}-\mathrm{Cm}$ ' and ' $\mathrm{Np}-\mathrm{Am}-\mathrm{Cm}$ ', were

\section{Acknowledgements}

The present research has been developed within the project 'PRACTICE' (Planning Re-thinked Ageing Cities Through Innovative Cellular Environments), 
financed under a cooperation framework between the Republic of Italy and the Kingdom of Sweden, and carried out by Sapienza - University of Rome and the Royal Institute of Technology of Stockholm, KTH. The authors desire also to thanks the Dynamic Makers team for their support.

\section{Appendix A. Detector Specifications}

In this paragraph Alphaino's electronics is discussed in depth. All components are listed in Table A.1. The repository for the project is available on GITHUB at https://github.com/bemxgm/Radon-Monitor.

The detector uses a photodiode as sensitive element. It is followed by a charge-to-voltage pre-amplifier and a multi-transimpedance amplification chain (with a numerical block for offset-compensation on the second amplifier) outside the sensitive area. A final analogical output and a signal-over-threshold output (driven by a comparator) are present on the board.

One of the operational amplifier is used to stabilize the voltage in a ring outside the photodiode area, as to avoid any current leakage. The multiamplification chain works at a mid-point voltage of $2.5 \mathrm{~V}$, and it is supplied by a low power voltage regulator. Between the pre-amplifier and the amplifying chain, a $0.033 \mu \mathrm{F}$ capacitor is placed to filter the signal, and to shape the pulse coming from the pre-amplifier. In reducing such capacitor, it is possible to get higher count rate with drawbacks for the amplification gain and noise on the final signal.

A voltage sum amplifier is used to regulate the output with respect to the common ground source, reducing the amplification gain at the expense of the signal noise. A voltage comparator is placed at the output to use the detector in Geiger-mode or time-over-threshold mode. The detector has a pin for the $2.5 \mathrm{~V}$ voltage source to use it as a reference, $V_{\text {ref }}$, for any ADC connected. The maximum $V_{\text {out }}$ is then $2.5 \mathrm{~V}$.

Figure A.2 shows the full-schematic. The complete board layout is shown in Figure A.1, right. 
Table A.1: Alphaino's components. The 'REF' code refers to the schematics in Figure A.2

\begin{tabular}{cll} 
Quantity & Description & REF \\
\hline 1 & ADR3425ARJZ-R7 & $\mathrm{U} 3$ \\
5 & $0.1 \mu \mathrm{F}, \pm 10 \%$ & $\mathrm{C} 1, \mathrm{C} 4, \mathrm{C} 5, \mathrm{C} 6, \mathrm{C} 8$ \\
1 & $\mathrm{LMP} 7721$ & $\mathrm{U} 2$ \\
1 & ILBB0805ER101V & $\mathrm{L} 2$ \\
1 & $100 \Omega, \pm 1 \%$ & $\mathrm{R} 3$ \\
1 & $\mathrm{MAX} 4478 \mathrm{ASD}+$ & $\mathrm{U} 1$ \\
1 & $2.2 \mathrm{M} \Omega, \pm 1 \%$ & $\mathrm{R} 6$ \\
1 & $470 \mathrm{k} \Omega, \pm 1 \%$ & $\mathrm{R} 9$ \\
2 & $100 \mathrm{pF}, \pm 2 \%$ & $\mathrm{C} 3, \mathrm{C} 7$ \\
7 & $1 \mathrm{k} \Omega, \pm 1 \%$ & $\mathrm{R} 2, \mathrm{R} 7, \mathrm{R} 11, \mathrm{R} 12, \mathrm{R} 14, \mathrm{R} 18, \mathrm{R} 19$ \\
1 & $\mathrm{LMV} 761 \mathrm{MFX}$ & $\mathrm{U} 4$ \\
1 & Trimmer $10 \mathrm{k} \Omega$ & $\mathrm{R} 10$ \\
1 & Photodiode, SLCD-61N5 & $\mathrm{U} 5$ \\
4 & Bridge $0 \Omega$ & $\mathrm{R} 5, \mathrm{R} 20, \mathrm{R} 21, \mathrm{R} 22$ \\
1 & $100 \mu \mathrm{F}, 6.3 \mathrm{~V}$ & $\mathrm{C} 13$ \\
1 & $33 \mathrm{nF}, \pm 5 \%$ & $\mathrm{C} 15$ \\
1 & $10 \Omega, \pm 1 \%$ & $\mathrm{R} 23$ \\
1 & $10 \mathrm{k} \Omega, \pm 1 \%$ & $\mathrm{R} 8$ \\
1 & $6.8 \mathrm{pF}, \pm 0.1 \mathrm{pF}$ & $\mathrm{C} 2$ \\
2 & $6.65 \mathrm{k} \Omega, \pm 1 \%$ & $\mathrm{R} 15, \mathrm{R} 16$ \\
1 & $\mathrm{HEADER}$ PIN & $\mathrm{J} 2$ \\
\hline & & \\
\hline
\end{tabular}




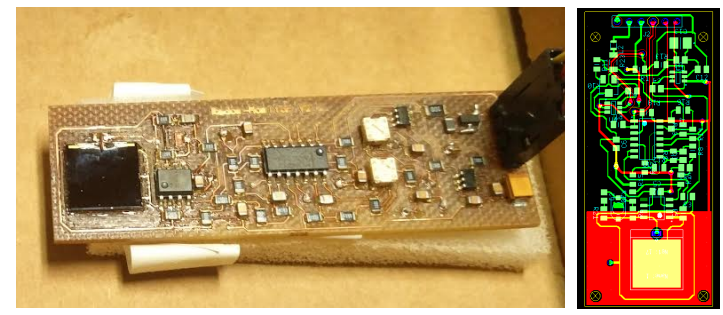

Figure A.1: Left: Alphaino detector. Right: board preview, full detailed file is available in the GITHUB repository.

The choice made in the amplification blocks layout is due to components' availability and cost on the market at the developing time. The most expensive part of the circuit is the photodiode. The total cost of all the other components the whole detector surface occupation (board included) is $2.7 \mathrm{~cm} \mathrm{x} 6.3 \mathrm{~cm}$, as shown in Figure A.1, left.

Even with a low pass filter integrated in the circuitry of Alphaino, it is strongly recommended to insulate the sensor from the external environment within a metal chamber e.g. made of simple aluminum foil or a conductive layer, connected to the ground. A closed environment is also necessary to avoid noise due to the visible light that can reach the photodiode surface, producing undesired signals. Such precaution can be combined in an electromagneticshielded (EMS) dark chamber, able to reduce light and electronic noise during operations.

The detector's performances depend upon the chosen photodiode, model SLCD-61N5 by Silonex, Inc. [8]. The expected system gain can be calculated as explained below. The oxide layer on the photodiode is obtained passivating the surface, and it can be considered around $\sim 90 \mathrm{~nm}$ thick. The Si-wafer width is $250 \mu \mathrm{m}$ and a $150 \mu \mathrm{m}$ gold plate is placed on the bottom of the photodiode and utilized as cathode. The anode is made by a $7 \mathrm{~mm} \times 1 \mathrm{~mm} \times 0.05 \mathrm{~mm}$ stripe of ITO (Indium Tin Oxide layer) on the P-region. Considering the $5 \mathrm{MeV}$ particle range of $5.566 \mathrm{mg} \mathrm{cm}^{-2}$ [18] in Amorphous Silicon (Figure A.3) at a density of 


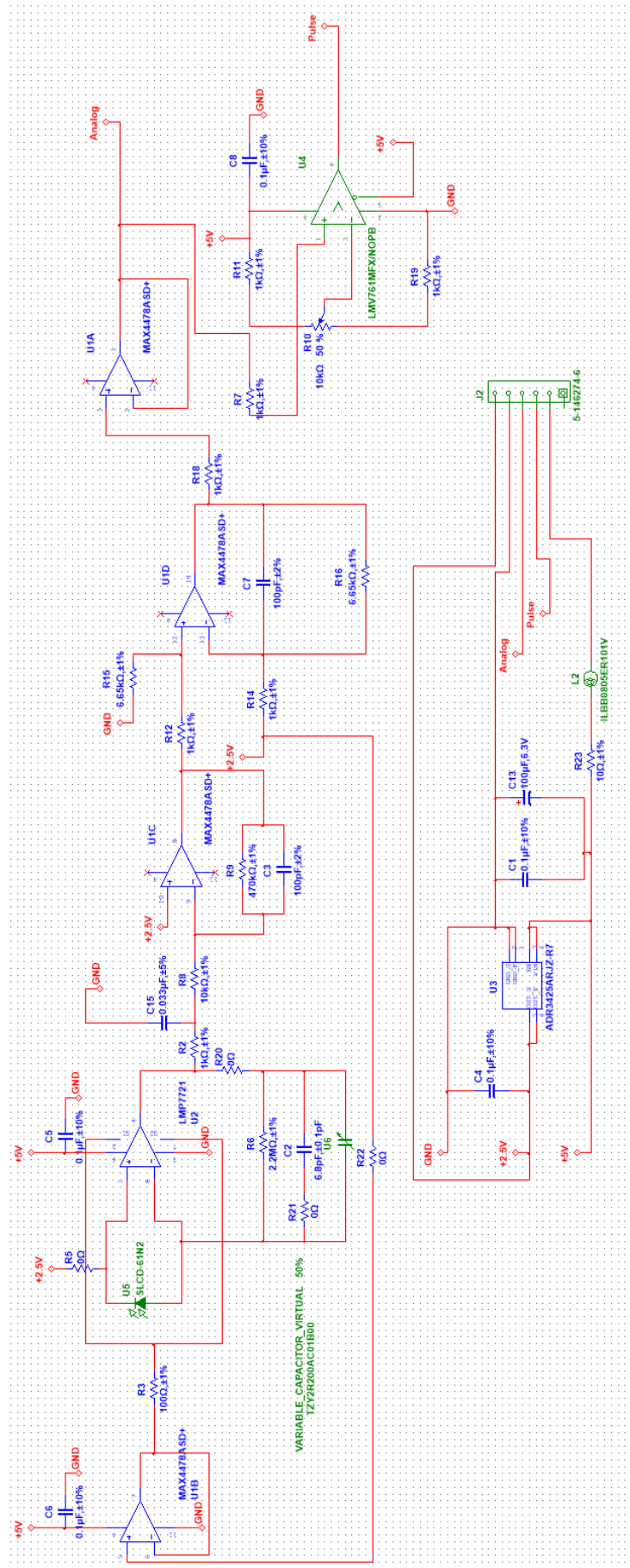

Figure A.2: Circuit scheme, full detailed scheme is available in the GITHUB repository. 


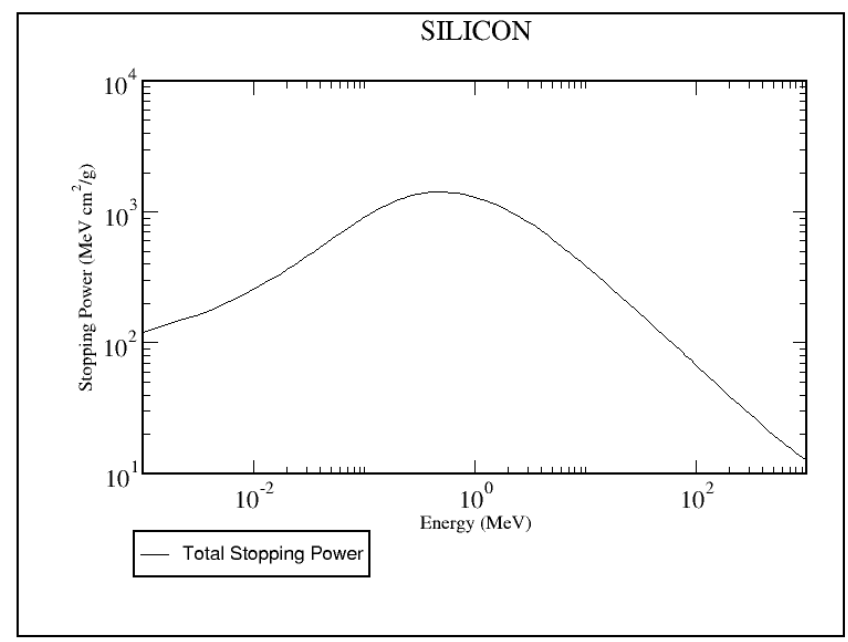

Figure A.3: Silicon stopping power from [18].

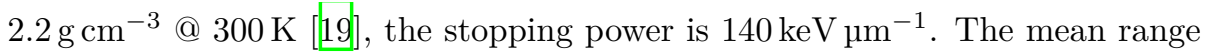
for a $5 \mathrm{MeV}$ alpha particle inside the photodiode is about $24.7 \mu \mathrm{m}$ [20]. As anticipated in Figure $\mathbb{1}$, the most significant part of the electric charges created by an alpha-track ionization in silicon is released and collected inside the active volume of the photodiode after the oxide layer.

The depletion depth is $W_{d}=4.93 \mu \mathrm{m}$, and can be calculated by Eq. A.1 considering the junction capacitance of $C_{j}=2 \mathrm{nF} @ 300 \mathrm{~K}$ in Eq. A.2,

$$
\begin{gathered}
W_{d}=\sqrt{2 \varepsilon_{S i} \varepsilon_{0} \mu \rho V_{T}} \\
C_{j}=\frac{\varepsilon_{S i} \varepsilon_{0} A}{W_{d}}
\end{gathered}
$$

where:

- $\varepsilon_{0}=8.854 \cdot 10^{-14} \mathrm{~F} \mathrm{~cm}^{-1}$ is the permittivity of free space, 
- $\varepsilon_{S i}=11.9$ is the silicon dielectric constant,

- $\mu=1400 \mathrm{~cm}^{2} \mathrm{~V}^{-1} \mathrm{~s}^{-1}$ is the mobility of the electrons @ $300 \mathrm{~K}$,

- $\rho=2.06 \Omega \mathrm{m}^{-1}$ is the resistivity of the photodiode's silicon,

- $V_{T}$ is the built-in voltage of the photodiode plus any eventual bias voltage,

- $A=93.6 \mathrm{~mm}^{2}$ is the photodiode area.

An energy deposition at the detector's sensitive volume of $5 \mathrm{MeV}$ by a single alpha particle can generate up to $1 * 400 * 000$ electrons by considering the average energy value for creating an ion-pair in silicon, $3.62 \mathrm{eV}$, namely [21]. Such electrons produce a total charge of $22.4 \mathrm{pC}$, causing a voltage drop of $0.112 \mathrm{mV}$ on a $2 \mathrm{nF}$ capacitor. A total gain of 22000 is necessary to get an output signal of $2.5 \mathrm{~V}$. Such gain is granted by many amplification loops at the expense of the measurements noise. Moreover, due to the big gain, the amplification circuit could be very sensitive to environmental electromagnetic noise and dark current: such an issue is solved by means of an electromagnetic-shielded chamber in which the detector is housed, even for its isolation from the external light.

\section{References}

[1] J. Bateman, Some new scintillator-photodiode detectors for high energy charged particles, Nuclear Instruments and Methods 67 (1) (1969) 93 102. doi:10.1016/0029-554X(69) 90546-1.

[2] R. Meijer, E. Bakkum, C. van Engelen, R. Kamermans, Light-particle detection with plastic scintillators coupled to a photodiode readout system, Nuclear Instruments and Methods in Physics Research Section A: Accelerators, Spectrometers, Detectors and Associated Equipment 240 (2) (1985) 333 - 337. doi:10.1016/0168-9002(85) 90645-X.

[3] T. Streil, R. Klinke, W. Birkholz, G. Just, New alpha radiation detection systems for radon and radon daughter monitoring, Radiation Measurements 25 (1) (1995) 621 - 622. doi:10.1016/1350-4487(95) 00201-0. 
[4] A. Ahmed, D. J. Walkey, N. G. Tarr, A CMOS integrated pulse mode alphaparticle counter for application in radon monitoring, IEEE Transactions on Nuclear Science 44 (3) (1997) 1425 - 1430. doi:10.1109/23.588588.

[5] P. Ashokkumar, B. K. Sahoo, A. Topkar, A. Raman, D. A. R. Babu, D. N. Sharma, Y. S. Mayya, An improved silicon pin diode based portable radon monitor, Indian Journal of Physics 87 (5) (2013) 471 - 477. doi:10.1007/ s12648-012-0243-7.

[6] L. Gugliermetti, D. A. Garcia, A cheap and third-age-friendly home device for monitoring indoor air quality, International journal of Environmental Science and Technology 10 (15) (2017) 185 -198. doi:10.1007/ s13762-017-1382-3.

[7] L. Gugliermetti, C. Galati, B. Mattoni, F. Nardecchia, F. Bisegna, Home smart grid device for energy saves and failure monitoring, Proceedings of the 15th International Conference on Environment and Electrical Engineering, IEEE (2015) 671 - 676 doi:10.1109/EEEIC.2015.7165245.

[8] Silonex INC., Slcd-61n5 photodiode datasheet (2018). URL http://www.farnell.com/datasheets/125183.pdf

[9] J. F. Briesmeister, MCNP - A general purpose Monte Carlo code for neutron and photon transport, Los Alamos National Laboratory, Report LA 12625, New Mexico, United States, 1993.

[10] D. Pelowitz, MCNPX User's Manual. Version 2.7.0, Los Alamos National Laboratory, Report LA-CP-11-00438, New Mexico, United States, 2011.

[11] Eckert \& Ziegler, Alpha Spectroscopy sources (2018). URL https://www.ezag.com/?id=183

[12] G. A. Marzo, A comparison of different peak shapes for deconvolution of alpha-particle spectra, Nuclear Instruments and Methods in Physics Research Section A: Accelerators, Spectrometers, Detectors and Associated Equipment 832 (2016) 191 - 201. 
doi:https://doi.org/10.1016/j.nima.2016.06.111.

URL http://www.sciencedirect.com/science/article/pii/ S0168900216306763

[13] G. Bortels, P. Collaers, Analytical function for fitting peaks in alphaparticle spectra from si detectors, International Journal of Radiation Applications and Instrumentation. Part A. Applied Radiation and Isotopes 38 (10) (1987) 831 - 837. doi:10.1016/0883-2889(87)90180-8.

[14] P. S. Foundation, Python language reference, version 3.6 (2018). URL http://www.python.org

[15] G. F. Knoll, Radiation detection and measurement; 2nd ed., Wiley, 1979, ISBN 0471815047.

[16] Laboratoire National Henri Becquerel, Commisariat à l'Énegie Atomique, Nucléide - Lara Library for gamma and alpha emissions (2018). URL http://www.nucleide.org/Laraweb/index.php

[17] Canberra Inc., PIPS Detectors (2018).

URL http://www. canberra.com/products/detectors/pdf/ passivated_pips_C39313a.pdf

[18] N. I. of Standard, P. M. L. Technology, Stopping-power and range tables for helium ions (2018).

URL https://physics.nist.gov/PhysRefData/Star/Text/ASTAR.html

[19] J. S. Custer, M. O. Thompson, D. C. Jacobson, J. M. Poate, S. Roorda, W. C. Sinke, F. Spaepen, Density of amorphous Si, Applied Physics Letters 64 (4) (1994) 437-439. doi:10.1063/1.111121.

[20] J. F. Ziegler, M. D. Ziegler, J. P. Biersack, SRIM - The stopping and range of ions in matter (2010), Nuclear Instruments and Methods in Physics Research B 268 (2010) 1818-1823. doi:10.1016/j.nimb.2010.02.091.

[21] G. F. Knoll, Radiation detection and measurement; 3rd ed., Wiley, 2000, ISBN 9780471073383. 